\title{
How to Optimise Extended Adjuvant Treatment with Neratinib for Patients with Early HER2+ Breast Cancer
}

\author{
Michael Untch · Miguel Martin · Michelino De Laurentiis • \\ Joseph Gligorov
}

Received: March 23, 2021 / Accepted: April 30, 2021 / Published online: May 31, 2021

(C) The Author(s) 2021

\section{ABSTRACT}

Over the last 20 years, treatment of patients with human epidermal growth factor receptor 2-positive (HER2+) early breast cancer has considerably improved. The development and addition of (neo)adjuvant trastuzumab to chemotherapy in patients with early HER2+ breast cancer (EHBC) has been shown to provide improvements in both disease-free survival (DFS) and overall survival, with some patients having a good prognosis being candidates for chemotherapy de-escalation strategies. However, despite such promising clinical outcomes, a significant proportion of patients still recur calling for the development of new preventive

\footnotetext{
M. Untch $(\bowtie)$

Breast Cancer Center, Helios Klinikum Berlin-Buch, Berlin, Germany

e-mail: michael.untch@helios-gesundheit.de

M. Martin

Oncology, Hospital General Universitario Gregorio

Maranon, Madrid, Spain
}

M. De Laurentiis

Breast Medical Oncology, Istituto Nazionale dei

Tumori IRCCS 'Fondazione Pascale', Napoli, Italy

J. Gligorov

Oncologie Médicale, Hôpital Tenon, Institut Universitaire de Cancérologie AP-HPOncologie Médicale, Hôpital Tenon, Institut Universitaire de Cancérologie AP-HP, Sorbonne Université, Inserm U938, Paris, France approaches. To this aim, the use of (neo)adjuvant trastuzumab for longer than one year or followed by lapatinib were tested without additional clinical improvement. Based on more recent advances, therapeutic strategies for patients with HER2+ tumours are now incorporating the use of newer (neo)adjuvant treatments, such as pertuzumab and trastuzumab emtansine, which have shown to further improve the invasive DFS (iDFS) benefit gained with trastuzumab. In this context, the tyrosine kinase inhibitor neratinib is approved in Europe for the extended adjuvant treatment of adult patients with early-stage hormone receptorpositive HER2+ breast cancer who completed adjuvant trastuzumab-based therapy less than one year ago. Clinical data have demonstrated that neratinib significantly improves iDFS when used for the total recommended duration of 12 months. This review paper provides an overview of the treatment of patients with EHBC, with a focus on the post-trastuzumab use of neratinib.

Keywords: Adjuvant treatment; Early breast cancer; Efficacy; Extended treatment; Human epidermal growth factor receptor 2-positive; Hormone receptor-positive; Neratinib; Tolerability; Trastuzumab; Tyrosine kinase inhibitor 


\section{Key Summary Points}

Why conduct this review?

Treatment of patients with early human epidermal growth factor receptor 2-positive (HER2+) breast cancer (EHBC) has improved over the last 20 years.

While the use of trastuzumab with chemotherapy in patients with EHBC improved disease-free and overall survival, a significant proportion of patients still recur calling for the development of new preventive approaches.

This review explores the use of the tyrosine kinase inhibitor neratinib, approved in Europe for the extended adjuvant treatment of adult patients with early-stage hormone receptor-positive $($ HR +$) /$ HER2 + breast cancer who completed adjuvant trastuzumab-based therapy less than one year ago.

What was learned from this review?

Neratinib represents a potential new efficacious solution to address the remaining risk of recurrence following trastuzumab-based (neo)adjuvant therapy in adult patients with early-stage hormone receptor-positive early stage HR+/HER2+ breast cancer and has demonstrated a manageable safety profile in the ExteNET study.

Extended adjuvant neratinib needs to be initiated soon after the end of trastuzumab therapy and maintained for the recommended 12 months to optimise treatment outcomes in patients with early-stage HR+/HER2+ breast cancer.

As some patients may initially experience episodes of diarrhoea in the first month of neratinib use, prophylactic strategies should be considered to support treatment adherence and help patients to remain on neratinib for the recommended 12 months.

\section{DIGITAL FEATURES}

This article is published with digital features, including a summary slide, to facilitate understanding of the article. To view digital features for this article go to https://doi.org/10.6084/ m9.figshare.14511963.

\section{INTRODUCTION}

Breast cancer is the most common type of cancer, the second leading cause of cancer death in women, and has a risk of recurrence following adjuvant treatment [1-3]. In approximately $15 \%-30 \%$ of invasive breast cancers, amplification of the human epidermal growth factor receptor 2 (HER2) gene or overexpression of the HER2 protein has been identified [3, 4].

Prior to the development of anti-HER2 agents, HER2+ breast cancer was considered to be a highly aggressive subtype with limited therapeutic options [5-7]. In particular, many HER2 + breast cancer recurrences were reported as distant metastases, with a cure no longer possible [1]. However, over the past 20 years, the development and subsequent availability of trastuzumab, a humanised monoclonal antibody that targets the extracellular domain of the HER2 protein, has significantly improved clinical outcomes for patients with early HER2+ breast cancer (EHBC) [1, 2, 8, 9]. The addition of trastuzumab to chemotherapy in EHBC can provide improvements in both disease-free survival (DFS) and overall survival (OS). Moreover, due to the importance and impact of HER2 blockade as a backbone strategy on the outcome of HER2+ cancers, some patients considered to have a good prognosis (small tumours and node-negative) may be candidates for chemotherapy de-escalation strategies.

However, despite these positive clinical outcomes, the use of trastuzumab for longer than one year in EHBC does not appear to further improve this therapeutic benefit, with disease recurrence reported in a proportion of patients [8]. In addition, strategies to combine the firstgeneration tyrosine kinase inhibitor (TKI) 
lapatinib to trastuzumab have failed to improve longer-term outcomes (ALLTO study) [10]

Available data suggest that approximately $15 \%-50 \%$ of patients with EHBC treated with adjuvant trastuzumab will go on to develop recurrent disease according to treatment strategies and initial stage of the disease $[2,8,21]$. Thus, the risk of disease recurrence remains significant for many patients and requires additional combination and/or sequencing of therapeutic strategies $[1,9,11,12]$. Newer adjuvant treatments for patients with EHBC include the monoclonal antibody pertuzumab and the TKI neratinib, along with trastuzumab emtansine (T-DM1), an antibody-drug conjugate of trastuzumab and the cytotoxic agent emtansine. This paper will examine the use of extended adjuvant treatment with neratinib for patients with EHBC, is based on previously conducted studies, and does not contain any new studies with human participants or animals performed by any of the authors.

\section{UNMET NEED IN THE TREATMENT OF PATIENTS WITH EHBC}

The treatment of patients with EHBC has seen an unprecedented advance with the development and regulatory approval of agents such as trastuzumab, pertuzumab, neratinib, and T-DM1. Trastuzumab was the first targeted agent indicated for the treatment of adult patients with EHBC [13]. Pertuzumab is indicated for use in combination with trastuzumab and chemotherapy as neoadjuvant treatment of adult patients with HER2+, locally advanced, inflammatory, or EHBC at high risk of recurrence and as adjuvant treatment of adult patients with EHBC at high risk of recurrence [14]. T-DM1 is indicated for the adjuvant treatment of adult patients with EHBC who have residual invasive disease in the breast and/or lymph nodes after neoadjuvant taxane-based and HER2-targeted therapy [15]. Neratinib is indicated for the extended adjuvant treatment of adult patients with hormone receptor-positive $(\mathrm{HR}+)$ EHBC who completed adjuvant trastuzumab-based therapy less than 1 year ago [16].
Data from a joint analysis of North Central Cancer Treatment Group NCCTG N9831 (Combination Chemotherapy With or Without Trastuzumab in Treating Women With HER2Overexpressing Breast Cancer) and the National Surgical Adjuvant Breast and Bowel Project NSABP B-31 (Doxorubicin and Cyclophosphamide Plus Paclitaxel With or Without Trastuzumab in Treating Women With NodePositive Breast Cancer That Overexpresses HER2), demonstrated that adjuvant trastuzumab significantly improved DFS by $40 \%$ (HR $0.60 ; 95 \%$ CI: $0.53,0.68 ; p<0.001)$ and increased the 10-year DFS rate from $62.2 \%$ to $73.7 \%$ in patients with EHBC [2]. Similarly, the Phase III BCIRG-006 study confirmed the longterm benefit of adjuvant trastuzumab in EHBC (particularly the regimen of doxorubicin and cyclophosphamide followed by paclitaxel and trastuzumab) and demonstrated improved DFS in high-risk subgroups of node-positive $(\mathrm{N}+)$ $[\geq 4 \mathrm{~N}+]$ patients with EHBC over 10 years of follow up [17].

While 12 months of adjuvant treatment with trastuzumab remains standard of care for selected low-risk adult patients with EHBC, different attempts to improve clinical outcomes by prolonging therapy duration beyond 1 year have failed, with disease recurrence reported in a proportion of patients. In the final analysis of the Phase III HERceptin Adjuvant (HERA) study, Cameron et al. reported that while adjuvant trastuzumab improved DFS, 2 years of treatment did not prevent further disease recurrence when compared with 1 year of treatment [8]. Alternative strategies have been tested to overcome limitations and possibly trastuzumab resistance by combining adjuvant trastuzumab with other treatments. In the Phase III ALTTO study, the addition of lapatinib to adjuvant trastuzumab therapy for 1 year did not significantly improve DFS compared with trastuzumab alone (HR $0.84 ; 97.5 \%$ CI: 0.70, $1.02 ; p=0.048$ ) and was also found to add toxicity [10]; patients treated with lapatinib reported more diarrhoea, cutaneous rash, and hepatic toxicity compared with trastuzumab alone. In the Phase III APHINITY study, the use of adjuvant trastuzumab in combination with pertuzumab and chemotherapy improved invasive DFS (iDFS) in 
patients with operable EHBC (HR 0.76; 95\% CI: $0.64,0.91$ ), with a 6 -year iDFS of $90.6 \%$ versus $87.8 \%$ without pertuzumab (difference of $2.8 \%$ ) [18]; however, the risk of recurrence remained, particularly for patients with $\mathrm{N}+$ disease (HR 0.72; 95\% CI: $0.59,0.87$ ), with a 6-year iDFS benefit of $4.5 \%$ ( $87.9 \%$ vs. $83.4 \%)$. The Phase III KATHERINE study of T-DM1 demonstrated that among patients with EHBC who had residual invasive disease after completion of neoadjuvant therapy, the risk of recurrence of invasive breast cancer or death was 50\% lower with adjuvant T-DM1 than with trastuzumab [12]; however, $11.7 \%$ of patients receiving T-DM1 had relapsed at 3 years. In addition, while there was a consistent benefit of T-DM1 across subgroups, the risk of recurrence remained substantial in patient populations with poor prognostic features, such as inoperable breast cancer at presentation, primary tumour stage of ypT4 at definitive surgery, and regional lymph node stage ypN3 at definitive surgery. A higher rate of treatment discontinuation due to adverse events was also reported with T-DM1 compared with trastuzumab $(18.0 \%$ vs. $2.1 \%$, respectively). Of note, it is important to remember that the majority of the patients included in the KATHERINE study had initial HER2+ and HR+ disease. Moreover, Untch et al. have identified that while distant metastases can be significantly avoided with T-DM1, central nervous system (CNS) first invasive recurrence appears more frequently with T-DM1 than with trastuzumab (numerically higher rate of CNS recurrence as first site of recurrence in the T-DM1 arm) due to the competing risks of these patients [19].

The achievement of pathologic complete response (pCR) (breast and axilla) is strongly prognostic for event-free survival and OS in early breast cancer [20]. Thus, patients achieving pCR can be expected to have good prognosis following neoadjuvant chemotherapy plus antiHER2+ therapy in patients with EHBC. However, Loibl et al. reported that while achieving pCR improved iDFS, this could not overcome the prognostic impact of baseline tumour size and nodal status which led to higher subsequent relapse compared with patients with small tumors at diagnosis and negative axillary nodes [21]. Such findings highlight that the risk of disease recurrence may still remain high (between 10 and 30\%) even in those patients who achieve a pCR. In fact, the traditional poor prognostic features at baseline remain determinant even after a pCR. Of note, Loibl et al. reported that there is no clear evidence that the relative impact of unfavourable features (cT3-4 or $\mathrm{N}+$ ) differs between patients with or without a pCR.

\section{USE OF NERATINIB IN THE EXTENDED ADJUVANT SETTING}

The current understanding of pathways causing breast cancer recurrence has been previously described in detail elsewhere [22]. In brief, breast cancers localised at the primary breast location which are treated early can still relapse due to the existence of cancer stem cells and transformation of cancer cells into a relatively aggressive phenotype. This recurrence of breast cancer is a major clinical concern and represents the principal cause of breast cancer-related deaths. A treatment strategy to further improve survival benefit with trastuzumab is needed.

For patients with HER2+ breast cancer, recurrence patterns may differ in $\mathrm{HR}+$ and $\mathrm{HR}-$ subgroups. Romond et al. reported relapse patterns according to HR status in patients with EHBC based on data from the two parallel registration clinical studies of trastuzumab plus paclitaxel after anthracycline chemotherapy for the adjuvant treatment of patients with HER2+ breast cancer (NSABP-B-31 and NCCTG-N9831) [23]. For patients with $\mathrm{HR}+$ disease, the recurrence risk continues to proportionally increase over time, while for patients with HR- disease, the relapse risk remains highest at an early stage after which it decreases proportionally over 7 years to a point where distant recurrence from breast cancer appears unlikely.

The concept of escalating adjuvant therapy for EHBC is that non-cross-resistant treatment administered in combination or sequentially with the standard trastuzumab-based adjuvant therapy may result in improved clinical outcomes [24]. Extended adjuvant therapy is one of 
the possible escalating approaches which prolongs the duration of adjuvant treatment.

Neratinib is an oral, small molecule TKI, targeting the HER2 (Fig. 1), HER4, and EGFR (HER1) kinases [16, 25, 26]. Neratinib binds at the ATP binding site and produces covalent modifications that are essentially irreversible [16]. Kourie et al. reported that neratinib is partially non-cross-resistant with trastuzumab [26].

The Phase III ExteNET study assessed the efficacy and safety of oral neratinib as extended adjuvant therapy in patients with EHBC who had completed adjuvant therapy with trastuzumab (including adjuvant or neoadjuvant strategy) [27]. The ExteNET study design is shown (Fig. 2). The primary efficacy outcome measure was iDFS, defined as the time between the dates of randomisation to the first occurrence of invasive recurrence (local/regional, ipsilateral, or contralateral breast cancer), distant recurrence, or death from any cause within 2 years and 28 days of follow-up in the intention-to-treat (ITT) population. The ExteNET study achieved its primary endpoint in the ITT population and demonstrated that neratinib for 12 months provided a clinically meaningful and

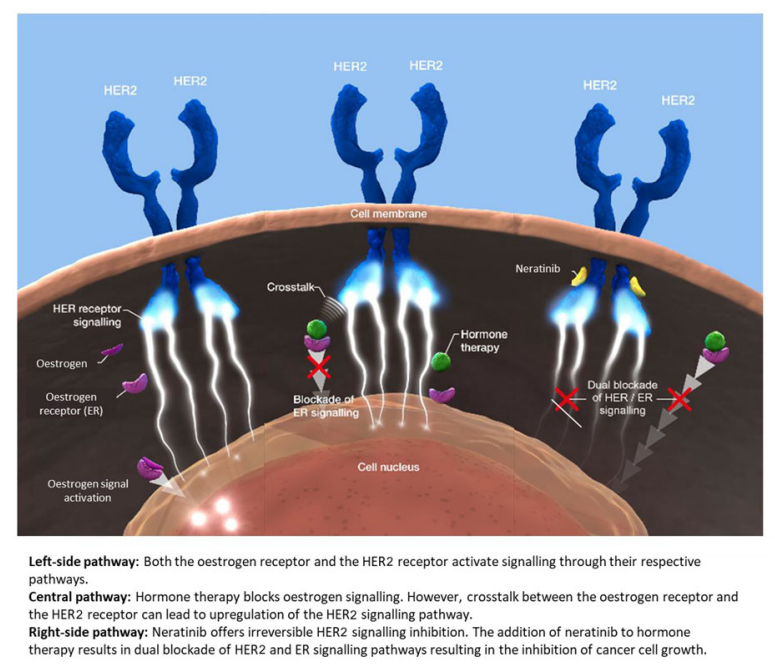

Fig. 1 Mechanism of action of neratinib on the HER2 pathway. ER oestrogen receptor; HER human epidermal growth factor receptor; HER2 human epidermal growth factor receptor 2. Figure reproduced with permission of Pierre Fabre statistically significant reduction in the risk of disease recurrence at 2 years when given after chemotherapy and trastuzumab-based adjuvant therapy [27]; 70 iDFS events were reported with neratinib compared with 109 iDFS events with placebo (stratified HR 0.67; 95\% CI: 0.50, 0.91; $p=0.0091$ ). The 2 -year iDFS rate was $93.9 \%$ (95\% CI: 92.4, 95.2) for patients treated with neratinib compared with $91.6 \%(90.0,93.0)$ for those who received placebo. This benefit was maintained at 5 years [28].

A pre-planned subgroup analysis of iDFS in the ExteNET study based on HR status showed that neratinib provided greater benefit to patients with $\mathrm{HR}+$ breast cancer at 2 years (HR 0.51; 95\% CI: 0.33, 0.77; $p=0.0013$ ) compared with HR- disease (HR 0.93; 95\% CI: 0.60, 1.43; $p=0.74)$ [27]. The higher benefit in HR+ disease was maintained at 5 years [28]. Of note, an additional prespecified subgroup analysis of iDFS demonstrated that women who had completed their last trastuzumab dose $>12$ months before starting the ExteNET study gained no significant benefit from extended adjuvant treatment with neratinib.

Further to combining the observations of superior benefit in patients who completed trastuzumab-based adjuvant treatment within 12 months and those with HR+ tumours, the European Medicines Agency requested an additional analysis in patients with HR+ tumours who started treatment $\leq 1$ year of completing trastuzumab $(\mathrm{HR}+/ \leq 1$-year population $)$ (Fig. 3; [29]). Among patients with HR+ breast cancer who started neratinib within 1 year of completing trastuzumab, there was an absolute iDFS benefit of $4.5 \%$ with neratinib after 2 years of follow-up (HR 0.49; 95\% CI: 0.30, 0.78; $p=0.002$ ). This treatment benefit with neratinib after 5 years of follow-up was durable with an absolute iDFS of $5.1 \%$ (HR $0.58 ; 95 \% \mathrm{CI}$ : $0.41,0.82 ; p=0.002$ ) and a $42 \%$ reduction in the relative risk of relapse. In the exploratory subset of patients with HR+ tumours who started treatment within 1 year of completing trastuzumab with no pCR after neoadjuvant therapy, there was an absolute iDFS benefit of $4.6 \%$ with neratinib at 2 years (HR $0.64 ; 95 \% \mathrm{CI}$ : $0.30,1.29$ ) and $7.4 \%$ at 5 years (HR $0.60 ; 95 \%$ 


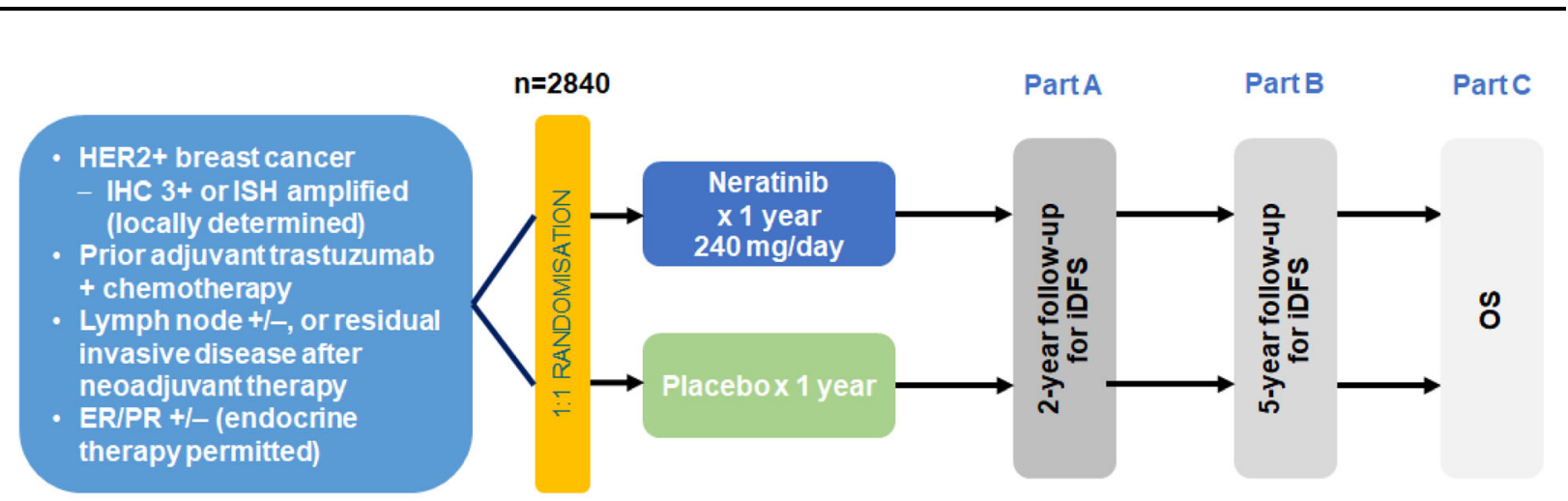

- Primary endpoint: iDFS

- Secondary endpoints: DFS-DCIS, time to distant recurrence, distant DFS, CNS metastases, OS, safety

- Additional analysis: biomarkers, health outcome assessment (FACT-B, EQ-5D)

- Stratified by: nodes 0,1-3 versus 4+, ER/PR status, concurrent versus sequential trastuzumab

Fig. 2 ExteNET study design [27]. CNS central nervous system; DCIS ductal carcinoma in situ; DFS disease-free survival; $E Q-5 D$ EuroQoL 5-dimension; $E R$ oestrogen receptor; $F A C T-B$ Functional Assessment of Cancer

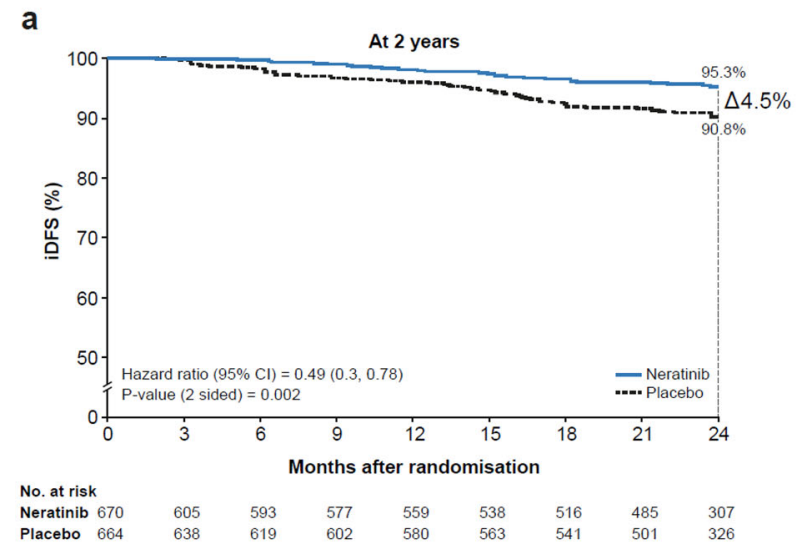

Fig. 3 iDFS for patients with HR+ tumours treated with neratinib $\leq 1$ year of completing trastuzumab at 2 years (a) and 5 years (b) (ExteNET study) [29]. Most patients (neratinib, 93\%; placebo, 95\%) were receiving concomitant

CI: $0.33,1.07)$, although these values were not statistically significant.

Final efficacy data from ExteNET in the $\mathrm{HR}+/$ s 1-year population post-trastuzumab were recently published [29]. Improvements in the neratinib arm were consistently observed across the study endpoints (iDFS, distant disease-free survival, OS). Descriptive analyses suggested that neratinib may be associated with
Therapy-Prostate; HER2+, human epidermal growth factor receptor 2-positive; $i D F S$ invasive disease-free survival; $I H C$ immunohistochemistry; $O S$ overall survival; $P R$ progesterone receptor

b

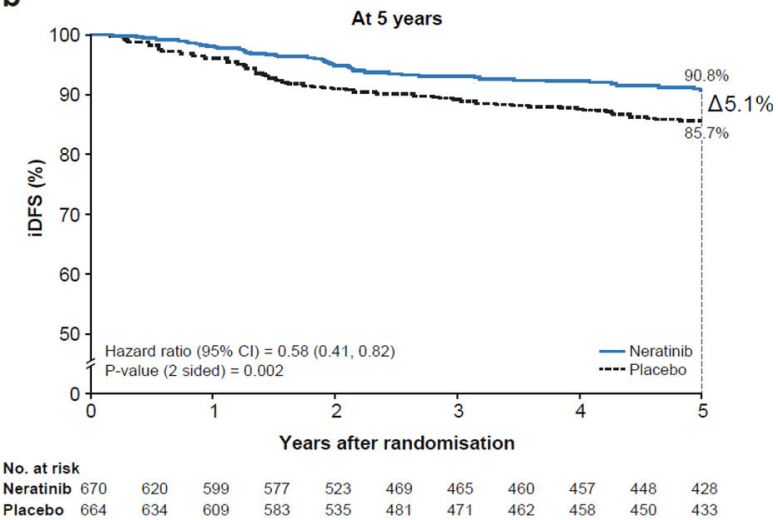

endocrine therapy at baseline. $H R+$ hormone receptorpositive; $i D F S$ invasive disease-free survival. Figures reproduced with permission from Clinical Breast Cancer

an absolute 8-year OS benefit of 2.1\% (HR 0.79; $95 \%$ CI: $0.55,1.13$ ) in the $\mathrm{HR}+/ \leq 1$-year population, which is clinically meaningful. Moreover, in the high-risk patient subgroup with residual disease after neoadjuvant therapy, the absolute 8-year OS benefit was 9.1\% (HR 0.47; $95 \%$ CI: $0.23,0.92$ ), and could be considered as being highly clinically meaningful, even if this was an exploratory analysis. 
Subsequent analysis of CNS recurrence data in a $\mathrm{HR}+/ \leq 1$-year population and in a subgroup of patients of clinical interest in the ExteNET study has also suggested that neratinib may reduce the risk of brain metastases [29]. Although the between-group differences did not reach statistical significance in these patient subgroups, the cumulative incidence of CNS recurrence was numerically lower in the neratinib arm at 5 years $(0.7 \%$ vs. $2.1 \%$ with placebo).

Diarrhoea was the most common treatment adverse event reported with neratinib in the ExteNET study $[27,28]$. In the absence of primary anti-diarrhoeal prophylactic measures, grade $1-2$, grade 3 , and grade 4 diarrhoea was reported in $55 \%, 40 \%$, and $<1 \%$ of patients who received neratinib (vs. $34 \%, 2 \%$, and $0 \%$ with placebo) [27]. Episodes of neratinib-associated diarrhoea typically appeared during the first weeks of treatment and lasted a median of 1-2 days per event regardless of severity. Of note, only one patient experienced grade 4 diarrhoea. Neratinib-related diarrhoea was not associated with any complications or long-term sequelae and did not appear to adversely affect clinical outcomes [30].

Escalating adjuvant therapy in EHBC can further decrease the risk of recurrence with number need to treat values of 10, 23, and 33 reported by the KATHERINE, ExteNET, and APHINITY studies $[12,18,28]$, respectively. As supported by international guidelines, neratinib represents a valuable solution to further reduce the risk of recurrence for patients completing (neo)adjuvant trastuzumab-based standard treatment [31-33].

\section{ADDITIONAL CONSIDERATIONS FOR PATIENT MANAGEMENT}

While the expansion of treatment options has improved the prognosis of patients with HER2+ breast cancer, the importance of therapy optimisation and patient adherence, along with side effect management and prophylaxis, to support treatment success should not be overlooked [34]. In a recent HER2+ breast cancer patient survey, it was reported that most (93\%) patients are concerned about disease recurrence, with many patients with HER $2+$ breast cancer stage I-IIIC (78\%) citing the fear of recurrence as their greatest concern. Patients with EHBC need to be made fully aware of the expected therapeutic benefit with any treatment, particularly where adherence to/persistence with treatment for a specific time frame appears to provide optimal therapeutic outcomes. For extended adjuvant therapy with neratinib following trastuzumab, patients need to start treatment soon after the end of trastuzumab therapy and persist with the full 12 months of neratinib in order to optimise clinical outcome [35].

\section{Management of Neratinib-Related Diarrhoea}

Diarrhoea is a class effect of the majority of TKIs [36]. In the ExteNET study, episodes of grade $\geq 2$ severity were short lived, while episodes of grade 3 diarrhoea were mainly reported in the first month of treatment [27, 30] (Fig. 4). Of note, antidiarrhoeal prophylaxis was not specified in the study protocol. The treatment of patients with diarrhoea in the ExteNET study was advised upon symptom manifestation. The median time to onset for grade $\geq 3$ events with neratinib was 8 days, and for any-grade diarrhoea was 2 days [30]. Grade 1 diarrhoea did not change appreciably over time, affecting about $30 \%$ of patients intermittently throughout the treatment period. The incidence of grade 3 diarrhoea decreased from $28.6 \%$ (month 1 ) to $11.2 \%$ (month 2 ) and was $\leq 6 \%$ after month 3 . While neratinib-related diarrhoea was not associated with any complications or long-term sequelae, diarrhoea led to neratinib dose reductions and discontinuation in $26 \%$ and $17 \%$ of patients, respectively, in the ExteNET study [27]. This finding suggests that targeted preventive management with antidiarrhoeal prophylaxis at an early stage during neratinib treatment is an appropriate strategy to mitigate episodes of diarrhoea and support treatment adherence [30].

Maintaining a positive balance between treatment benefit and adverse events is of major importance in early-stage breast cancer. The 


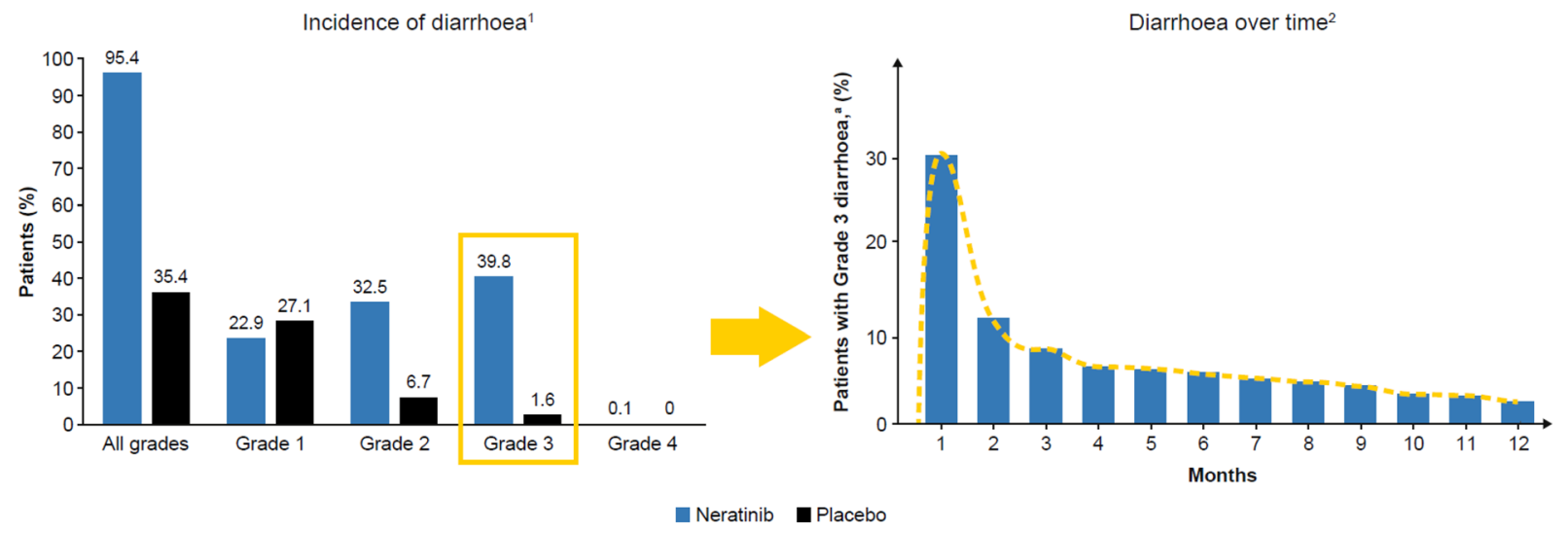

Fig. 4 Diarrhoea over time with neratinib without systematic prophylaxis (ExteNET study) $[27,30)] .{ }^{a}$ One patient in the ExteNET study experienced grade 4 diarrhoea

open-label, sequential-cohort, Phase II CONTROL study (NCT02400476) is investigating the effect of different anti-diarrhoeal strategies on neratinib-associated diarrhoea in patients with EHBC who completed previous trastuzumabbased adjuvant therapy less than one year ago, including pertuzumab and T-DM1. Initial cohorts included loperamide prophylaxis alone or with budesonide (a locally acting corticosteroid used for inflammatory gastrointestinal conditions) or colestipol (a bile-acid sequestrant) combined from the treatment start with neratinib $240 \mathrm{mg} /$ day full dose. Two additional cohorts investigating modified neratinib doseescalation regimens were subsequently added. The weekly dose-escalation regimen (DE1) starts with neratinib administered at $120 \mathrm{mg}$ daily during week 1 , escalated to $160 \mathrm{mg}$ daily during week 2 , and to $240 \mathrm{mg}$ daily starting from week 3 up to 1 year; the bi-weekly dose-escalation (DE2) starts with $160 \mathrm{mg}$ daily of neratinib for the first 2 weeks, escalated to $200 \mathrm{mg}$ during week 3 and 4 to reach the full dose of $240 \mathrm{mg}$ of neratinib from week 5 . In both dose-escalation cohorts, loperamide is administered as needed to maximum dose of $16 \mathrm{mg} /$ day. The primary endpoint of the CONTROL study is the incidence of grade 3 diarrhoea [37]. Final results from the first 5 cohorts, including the DE1 regimen, recently demonstrated a lower incidence of grade 3 diarrhoea compared with ExteNET in all cohorts (loperamide 31\%, budesonide plus loperamide 28\%, colestipol plus loperamide $21 \%$, colestipol plus as-needed loperamide $33 \%$, neratinib weekly dose escalation 8\%) [38]. Diarrhoea-related discontinuations were $20 \%$ with loperamide use, $11 \%$ with budesonide plus loperamide, $4 \%$ with colestipol plus loperamide, $8 \%$ with colestipol plus asneeded loperamide, while the lowest level (3\%) was reported with DE1. In addition, the median cumulative duration of grade 3 diarrhoea was low (2.5 days) with DE1. Decreases in healthrelated quality of life did not cross the clinically important threshold [37]. While the investigation of DE2 is still ongoing, the future adoption of neratinib dose-escalation strategies might reduce the incidence, severity, and duration of neratinib-associated diarrhoea in the CONTROL study compared with the ExteNET study [38].

In addition, special attention should be given to patient nutrition and diet when receiving neratinib in order to avoid and minimise diarrhoea. Eating small frequent meals, drinking more clear liquids (8-12 glasses per day, such as water, clear juices, broth, weak decaffeinated tea, and caffeine-free sports/soft drinks), and opting for easily digested foods (low-residue diet, which includes foods such as bananas, rice, apple sauce, and toast) should be advised. The patient should also refrain from consuming caffeine, alcohol, dairy, fat, fibre, orange juice, grapefruit juice, prune juice, and spicy foods. 


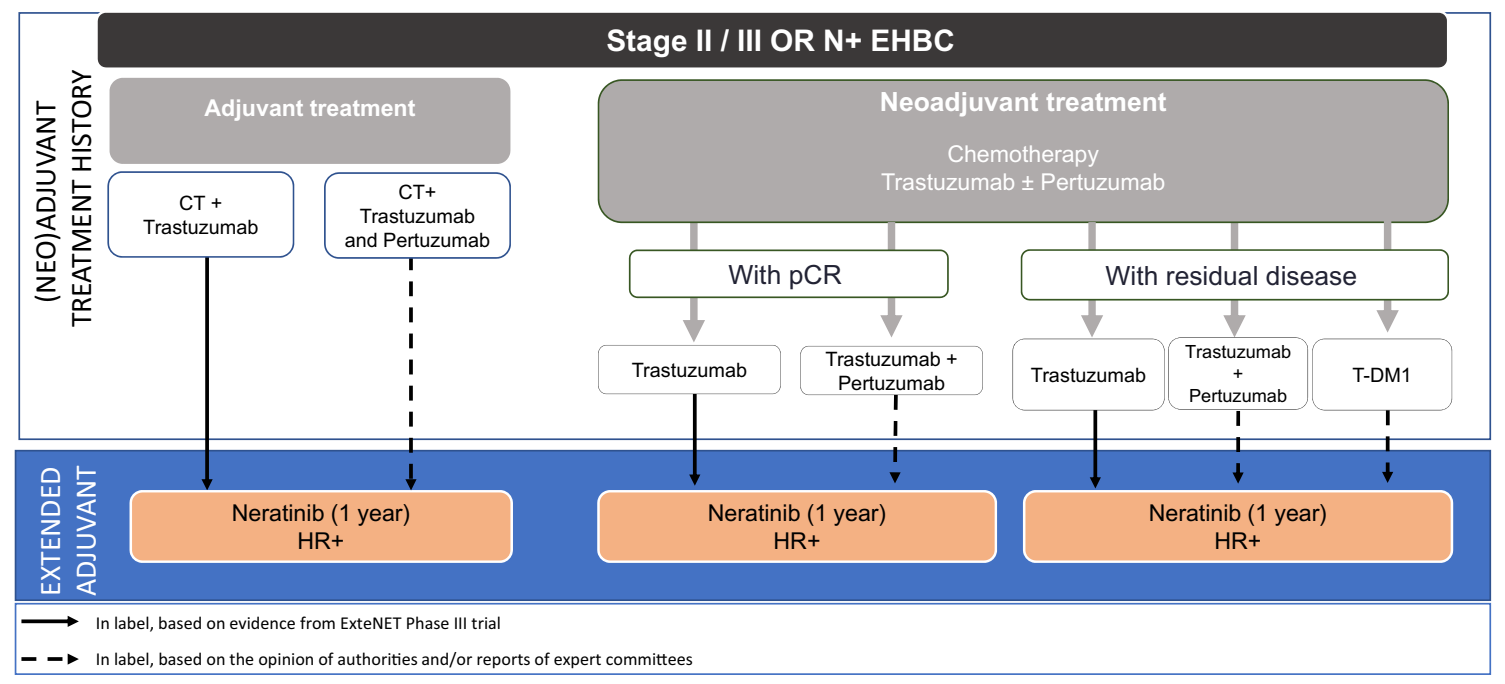

Fig. 5 Extended adjuvant therapy with neratinib in EHBC: possible clinical scenarios [13-16]. EHBC early HER2+ breast cancer; HER2+ human epidermal growth

\section{The Need for Effective Patient-Physician Communication}

A robust physician-patient alliance remains important to optimise treatment outcomes. This includes the active contribution of the patient in therapy, given that patients with HER2+ breast cancer are highly engaged in treatment planning [34]. Patient-physician communication should provide the opportunity to discuss available treatment options and supporting evidence, along with expected outcomes. In addition, the patient should be fully informed of any potential treatment side effects (e.g., diarrhoea) and a robust plan developed to effectively manage them at an early stage.

\section{REAL-WORLD TREATMENT PATTERNS IN PATIENTS WITH EHBC USING NERATINIB AS EXTENDED ADJUVANT TREATMENT}

The current treatment landscape for patients with EHBC has evolved significantly in the last few years and the clinical practice in some countries may currently include the use of two additional HER2-directed agents (pertuzumab factor receptor 2-positive; $H R$ hormone receptor; $N$ lymph nodes/nodal status; $p C R$ pathological complete response; $T-D M 1$ trastuzumab emtansine

and trastuzumab emtansine) as trastuzumabbased therapy, substantially differing from the patient population eligible for extended adjuvant treatment with neratinib in ExteNET study. Despite the efficacy of neratinib in this setting remaining unknown, neratinib might represent a potentially non-cross resistant therapy to prevent recurrences in high-risk patients even after treatment with pertuzumab and/or trastuzumab emtansine.

Taking into account these therapeutic advances and acknowledging the different level of evidence for neratinib use in the different depicted treatment patterns, Fig. 5 aims to summarise the possible clinical scenarios for extended adjuvant consideration, upon integration with existing treatment guidelines and careful benefit/risk evaluation on an individual patient basis (Fig. 5).

\section{SUMMARY}

Over the last two decades, EHBCs have transitioned from a disease with a poor prognosis to one which is more favourable. In patients with EHBC, initial disease stage and/or response to neoadjuvant treatment are relevant prognostic factors to guide the choice of anti-HER2 
strategies. However, an unmet medical need remains for some patients with HER2+ breast cancers in the adjuvant setting given that approximately one third of them remain at risk of disease recurrence and metastasis following optimal adjuvant therapy. While post-neoadjuvant T-DM1 is recommended and approved for the treatment of patients with EHBC who have residual invasive disease based on data from the KATHERINE study, disease progression following this treatment approach remains a challenge. In addition, the risk of disease recurrence may remain high even in those patients who initially achieve pCR. Neratinib represents a potential new efficacious solution to address the remaining risk of recurrence following trastuzumab-based (neo)adjuvant therapy and has demonstrated a manageable safety profile in the ExteNET study. It is important to note that extended adjuvant neratinib needs to be initiated soon after the end of trastuzumab therapy and maintained for the recommended 12 months to optimise treatment outcomes in patients with $\mathrm{HR}+/ \mathrm{EHBC}$. As some patients may initially experience episodes of diarrhoea in the first month of neratinib use, prophylactic strategies, and a dose-escalation strategy may be required to support treatment adherence. The increasing clinical experience with neratinib in the real-world clinical setting, together with ongoing research efforts on diarrhoea prevention and management, will serve to further improve our knowledge on its safety profile in the extended adjuvant treatment setting.

\section{ACKNOWLEDGEMENTS}

Funding. Third-party medical writing support and the Journal's Rapid Service Fee and Open Access fee for this manuscript was funded by Pierre-Fabre.

Medical Writing, Editorial, and Other Assistance. Third-party medical writing support was provided by Matthew Joynson of Springer Healthcare Ltd.
Authorship. All named authors meet the International Committee of Medical Journal Editors (ICMJE) criteria for authorship for this article, take responsibility for the integrity of the work as a whole, and have given their approval for this version to be published.

Authorship Contributions. Michael Untch, Miguel Martin, Michelino De Laurentiis, and Joseph Gligorov all contributed to the writing, editing, and final review of this manuscript.

Prior Publication. This manuscript is based on work that has been previously presented/ published.

Disclosures. Michael Untch declares that he has had fees paid directly to his institution for advisory boards, speaker honoraria, and travel support from AbbVie, Amgen, AstraZeneca, Celgene, Daiichi Sankyo, Eisai, Lilly Germany, Lilly International, Merck, MSD Mundipharma, Myriad Genetics, Novartis, Odonate, Pierre Fabre, Pfizer, PUMA Biotechnology, F. HoffmanLa Roche, Sanofi Aventis, and TEVA Pharmaceuticals.

Miguel Martin declares that he has received research grants from Roche, PUMA, and Novartis, consulting/advisory fees from AstraZeneca, Amgen, Taiho Oncology, Roche/Genentech, Novartis, PharmaMar, Eli Lilly, PUMA, Daiichi Sankyo, and Pfizer, and Speakers' honoraria from AstraZeneca, Amgen, Roche/Genentech, Novartis, and Pfizer.

Michelino De Laurentiis declares that he has received fees for speakers' honoraria/advisory role from Pfizer, Novartis, Roche, Celgene, AstraZeneca, Eisai, Eli Lilly, Amgen, MSD, Pierre Fabre, Daiichi Sankyo, Seagen, and PUMA Biotechnology.

Joseph Gligorov declares that he has received grants, fees and non-financial support from Eisai, Genomic Health, Roche/Genentech, and Pfizer, fees and non-financial support from Daiichi Sankyo, MSD, and Novartis, and fees from Ipsen, Macrogenics, Onxeo, and Pierre Fabre.

Compliance with Ethics Guidelines. This article is based on previously conducted studies 
and does not contain any studies with human participants or animals performed by any of the authors.

Data Availability. All data and references mentioned in this manuscript are from publicly available sources. Data sharing is not applicable to this article, as no data sets were generated or analysed during the current study.

Open Access. This article is licensed under a Creative Commons Attribution-NonCommercial 4.0 International License, which permits any non-commercial use, sharing, adaptation, distribution and reproduction in any medium or format, as long as you give appropriate credit to the original author(s) and the source, provide a link to the Creative Commons licence, and indicate if changes were made. The images or other third party material in this article are included in the article's Creative Commons licence, unless indicated otherwise in a credit line to the material. If material is not included in the article's Creative Commons licence and your intended use is not permitted by statutory regulation or exceeds the permitted use, you will need to obtain permission directly from the copyright holder. To view a copy of this licence, visit http://creativecommons.org/licenses/by$\mathrm{nc} / 4.0 /$.

\section{REFERENCES}

1. Piccart-Gebhart MJ, Procter M, Leyland-Jones B, et al. Trastuzumab after adjuvant chemotherapy in HER2-positive breast cancer. $N$ Engl J Med. 2005;353:1659-72.

2. Perez EA, Romond EH, Suman VJ, et al. Trastuzumab plus adjuvant chemotherapy for human epidermal growth factor receptor 2-positive breast cancer: planned joint analysis of overall survival from NSABP B-31 and NCCTG N9831. J Clin Oncol. 2014;32:3744-52.

3. Miles J, White Y. Neratinib for the treatment of early-stage HER2-positive breast cancer. J Adv Pract Oncol. 2018;9:750-4.

4. Slamon DJ, Godolphin W, Jones LA, et al. Studies of the HER-2/neu proto-oncogene in human breast and ovarian cancer. Science. 1989;244:707-12.
5. Wolff AC, Hammond ME, Hicks DG, et al. Recommendations for human epidermal growth factor receptor 2 testing in breast cancer: American Society of Clinical Oncology/College of American Pathologists clinical practice guideline update. J Clin Oncol. 2013;31:3997-4013.

6. Sorlie T, Tibshirani R, Parker J, et al. Repeated observation of breast tumor subtypes in independent gene expression data sets. Proc Natl Acad Sci U S A. $2003 ; 100: 8418-23$.

7. Chia SK, Norris B, Speers C, et al. Human epidermal growth factor receptor 2 overexpression as a prognostic factor in a large tissue microarray series of node-negative breast cancers. J Clin Oncol. 2008;26:5697-704.

8. Cameron D, Piccart-Gebhart MJ, Gelber RD, et al. for the Herceptin Adjuvant (HERA) Trial Study Team. 11 years' follow-up of trastuzumab after adjuvant chemotherapy in HER2-positive early breast cancer: final analysis of the HERceptin Adjuvant (HERA) trial. Lancet. 2017;389:1195-205.

9. von Minckwitz G, Procter M, de Azambuja E, et al. Adjuvant pertuzumab and trastuzumab in early HER2-positive breast cancer. $\mathrm{N}$ Engl J Med. 2017;377:122-31.

10. Moreno-Aspitia A, Holmes EM, Jackisch C, et al; ALTTO Steering Committee and Investigators. Updated results from the international phase III ALTTO trial (BIG 2-06/Alliance N063D). Eur J Cancer. 2021;148:287-96.

11. Gianni L, Pienkowski T, Im YH, et al. Efficacy and safety of neoadjuvant pertuzumab and trastuzumab in women with locally advanced, inflammatory, or early HER2-positive breast cancer (NeoSphere): a randomised multicentre, open-label, phase 2 trial. Lancet Oncol. 2012;13:25-32.

12. von Minckwitz G, Huang CS, Mano MS, et al. Trastuzumab emtansine for residual invasive HER2positive breast cancer. N Engl J Med. 2019;380: 617-28.

13. Herceptin SPC. 2020. Available at: https://www. ema.europa.eu/en/documents/productinformation/herceptin-epar-product-information en.pdf. (Accessed 11 Sept 2020).

14. Perjeta SPC. 2020. Available at: https://www.ema. europa.eu/en/documents/product-information/ perjeta-epar-product-information_en.pdf. (Accessed 11 Sept 2020).

15. Kadcyla SPC. 2020. Available at: https://www.ema. europa.eu/en/documents/product-information/ kadcyla-epar-product-information_en.pdf. (Accessed 11 Sept 2020). 
16. Nerlynx SPC. 2020. Available at: https://www.ema. europa.eu/en/documents/product-information/ nerlynx-epar-product-information_en.pdf. (Accessed 11 Sept 2020).

17. Slamon DJ, Eiermann W, Robert NJ, et al, on behalf of the BCIRG-006 Investigators. Ten year follow-up of BCIRG-006 comparing doxorubicin plus cyclophosphamide followed by docetaxel $(\mathrm{AC} \rightarrow \mathrm{T})$ with doxorubicin plus cyclophosphamide followed by docetaxel and trastuzumab $(\mathrm{AC} \rightarrow \mathrm{TH})$ with docetaxel, carboplatin and trastuzumab (TCH) in HER2+ early breast cancer. Cancer Res. 2016;76(4 Suppl):S5-04.

18. Piccart $M$, Procter $M$, Fumagalli D, et al. Interim overall survival analysis of APHINITY (BIG 4-11): A randomized multicenter, double-blind, placebocontrolled trial comparing chemotherapy plus trastuzumab plus pertuzumab versus chemotherapy plus trastuzumab plus placebo as adjuvant therapy in patients with operable HER2-positive early breast cancer. Cancer Res. 2020;80(4 Suppl):Abstract GS104 .

19 Untch M, Geyer CE Jr, Huang CS, et al. Peripheral neuropathy (PN), thrombocytopenia (TCP) and central nervous system (CNS) recurrence: An update of the phase III KATHERINE trial of post-neoadjuvant trastuzumab emtansine (T-DM1) or trastuzu$\mathrm{mab}(\mathrm{H})$ in patients (pts) with residual invasive HER2-positive breast cancer (BC). Ann Oncol. 2019;30(9):183.

20. Cortazar P, Zhang L, Untch M, et al. Pathological complete response and long-term clinical benefit in breast cancer: the CTNeoBC pooled analysis. Lancet. $2014 ; 384: 164-72$.

21. Loibl S, Untch M, Buyse M, et al. Pathologic complete response (pCR) and prognosis following neoadjuvant chemotherapy plus anti-HER2 therapy of HER2-positive early breast cancer (EBC). Cancer Res. 2020;80(4):2.

22. Ahmad A. Pathways to breast cancer recurrence. Isrn Oncol. 2013;2013:290568.

23. Romond EH, Suman V, Jeong J-H, et al. Trastuzumab plus adjuvant chemotherapy for HER2positive breast cancer: Final planned joint analysis of overall survival from NSABP B-31 and NCCTG N9831. Cancer Res. 2012;72(24Sup):Abstract S5-5.

24. Pegram M. Can we circumvent resistance to ErbB2targeted agents by targeting novel pathways? Clin Breast Cancer. 2008;8(Suppl 3):S121-30.

25. Baselga J, Coleman RE, Cortés J, Janni W. Advances in the management of HER2-positive early breast cancer. Crit Rev Oncol Hematol. 2017;119:113-22.
26. Kourie HR, El Rassy E, Clatot F, de Azambuja E, Lambertini M. Emerging treatments for HER2-positive early-stage breast cancer: focus on neratinib. OncoTargets Ther. 2017;10:3363-72.

27. Chan A, Delaloge S, Holmes FA, et al; ExteNET Study Group. Neratinib after trastuzumab-based adjuvant therapy in patients with HER2-positive breast cancer (ExteNET): a multicentre, randomised, double-blind, placebo-controlled Phase 3 trial. Lancet Oncol. 2016;17:367-77.

28. Martin M, Holmes FA, Ejlertsen B, et al. Neratinib after trastuzumab-based adjuvant therapy in HER2positive breast cancer (ExteNET): 5-year analysis of a randomised, double-blind, placebo-controlled, phase 3 trial. Lancet Oncol. 2017;18:1688-700.

29. Chan A, Moy B, Mansi J, et al; ExteNET Study Group. Final efficacy results of neratinib in HER2positive hormone receptor-positive early-stage breast cancer from the phase III ExteNET trial. Clin Breast Cancer. 2021;21:80-91.e1.

30. Mortimer J, Di Palma J, Schmid K, Ye Y, Jahanzeb M. Patterns of occurrence and implications of neratinib-associated diarrhea in patients with HER2positive breast cancer: analyses from the randomized phase III ExteNET trial. Breast Cancer Res. 2019;21:32.

31. Cardoso F, Kyriakides S, Ohno S, et al. Early breast cancer: ESMO clinical practice guidelines for diagnosis, treatment and follow-up. Ann Oncol. 2019;30:1194-220.

32. Gradishar WJ, Anderson BO, Abraham J, et al. Breast cancer, version 32020, NCCN clinical practice guidelines in oncology. J Natl Compr Canc Netw. 2020;18:452-78.

33. Denduluri N, Chavez-MacGregor M, Telli ML, et al. Selection of optimal adjuvant chemotherapy and targeted therapy for early breast cancer: ASCO clinical practice guideline focused update. J Clin Oncol. 2018;36:2433-43.

34. Jenkins M. Patient perspectives on HER2+ breast cancer recurrence: results from an online patient survey. Cancer Res. 2019;79(4 Suppl):Abstract P514-03.

35. Martin M, Gnant MI, Ejlertsen B, et al. Impact of treatment duration of extended adjuvant therapy with neratinib in early stage HER2+ HR+ breast cancer after trastuzumab-based therapy on patient outcomes. Ann Oncol. 2020;31(S2):Abstract 83P.

36. Hartmann JT, Haap M, Kopp HG, Lipp HP. Tyrosine kinase inhibitors - a review on pharmacology, metabolism and side effects. Curr Drug Metab. 2009; 10:470-81. 
37. Barcenas $\mathrm{CH}$, Hurvitz SA, Di Palma JA, et al. Improved tolerability of neratinib in patients with HER2-positive early-stage breast cancer: the CONTROL trial. Ann Oncol. 2020;31:1223-30.

38. Ruiz-Borrego M, Chan A, Marx G, et al; the CONTROL Investigators. Bringing diarrhea under
CONTROL: dose escalation reduces neratinib-associated diarrhea and improves tolerability in HER2positive early-stage breast cancer. Abstract PS13-20. Presented at San Antonio Breast Cancer Symposium, 8-11. 2020. 